\title{
Tank Mixing Fungicides for Effectiveness Against Eastern Filbert Blight of Hazelnut
}

\author{
J. W. Pscheidt, ${ }^{\dagger}$ S. Heckert, and S. A. Cluskey, Department of Botany \& Plant Pathology, Oregon State University, Corvallis 97331
}

\begin{abstract}
Hazelnut (Corylus avellana) production in Oregon primarily occurs on cultivars susceptible to Anisogramma anomala, the causal agent of eastern filbert blight (EFB). Management of EFB involves planting resistant cultivars, removal of cankered limbs, and the application of fungicides. Tank mixes of demethylation-inhibiting (DMI; Fungicide Resistance Action Committee [FRAC] group 3) or quinone outside inhibitor (QoI; FRAC group 11) fungicides with chlorothalonil (FRAC group M5) at full or reduced rates were evaluated for effectiveness against $A$. anomala. The use of chlorothalonil in a mix with a DMI or QoI fungicide was an effective treatment for EFB even if each component of the mix was at half the labeled rate. Different liquid or dry formulations of chlorothalonil were equally effective in a tank mix for

EFB control. The combination of propiconazole (FRAC group 3) tank mixed with trifloxystrobin (FRAC group 11) was not effective, whereas trees treated with propiconazole tank mixed with pyraclostrobin (FRAC group 11) resulted in significantly fewer EFB cankers compared with nontreated trees. When using tank mixes for EFB management, DMI fungicides should remain at full rates while mixing with a half-rate of chlorothalonil. In contrast, QoI fungicides and chlorothalonil could both be used at half-rates and still maintain acceptable EFB control. Tank mixing chlorothalonil with fungicides at risk of resistance development can help maintain consistent EFB control and should help prevent or delay the emergence of fungicide-resistant $A$. anomala isolates.
\end{abstract}

Most of the hazelnut (Corylus avellana L.) production in Oregon of 38,000 tons in 2014, for a farm gate value of $\$ 130$ million, came from cultivars susceptible to Anisogramma anomala (Peck) E. Müll. 1962 (Diaporthales), the causal agent of eastern filbert blight (EFB) (Oregon Department of Agriculture 2015). The Hazelnut Bargaining Association negotiated a record-setting minimum price of $\$ 3.75 / \mathrm{kg}$ $(\$ 1.70 / \mathrm{lb}$ ) for hazelnut in 2014 , in contrast to the low $\$ 0.66 / \mathrm{kg}$ $(\$ 0.30 / \mathrm{lb})$ in 1993 , when EFB was emerging as a major industry problem (Johnson et al. 1996). Management of EFB involves planting resistant cultivars (cash cost of $\$ 3,479$ to $5,360 /$ ha $[\$ 1,408$ to 2,169 / acre] to establish a new orchard), removal of cankered limbs (over $\$ 370 /$ ha [ $\$ 150 /$ acre]), and the application of fungicides ( $\$ 45$ to $89 / \mathrm{ha}$ [\$18 to 36/acre]) during bud break and early shoot growth (Heckert et al. 2014; Johnson et al. 1996; Miller et al. 2013; Pscheidt and Ocamb 2017). Encouraging growers to adopt new production practices for management of EFB was difficult when prices were low in the early 1990s but these practices have become common as nut prices increased.

Many fungicide active ingredients are registered on hazelnut for use against EFB but only represent five different Fungicide Resistance Action Committee (FRAC) groups, including demethylationinhibiting (DMI) fungicides (FRAC group 3) (Johnson et al. 1993; Pscheidt and Cluskey 2001), quinone outside inhibitors (QoI) (FRAC group 11), and succinate dehydrogenase inhibitors (FRAC group 7) (Pscheidt et al. 2017), as well as copper-based (FRAC group M1) and chlorothalonil (FRAC group M5) (Pscheidt and Cluskey 2001) fungicides. Prepackaged combinations of two or more of these active ingredients are also registered (Pscheidt and Ocamb 2017). In

${ }^{\dagger}$ Corresponding author: J. W. Pscheidt;

E-mail: pscheidj@science.oregonstate.edu

Funding: This research was regularly supported, in part, by the Oregon Hazelnut Commission, which is a Commodity Commission and agency of the State of Oregon under the Oregon Department of Agriculture. Additional support was provided in part by the following manufacturers through either small monetary donations or fungicide product donations: ADAMA, Arysta, BASF, Bayer, Sipcam Agro USA, Syngenta, United Phosphorus Inc., and Valent USA.

Accepted for publication 12 November 2017.

(c) 2018 The American Phytopathological Society total, four fungicide applications are recommended starting at bud break (early to mid-March) and continuing at regular intervals (every 2 weeks, depending on the weather and material used) over an 8week period (until early May) to prevent $A$. anomala infection (Pscheidt and Cluskey 2001; Pscheidt and Ocamb 2017; Stone et al. 1992).

Development of A. anomala resistant to fungicides has not been documented or even suspected. The likelihood of resistance development is low, given the lack of a conidial repeating stage and a 2-year or more generation time of this fungus (Gottwald and Cameron 1979). However, resistance management through the use of multiple active ingredients is prudent, given that sexual recombination occurs each generation (Gottwald and Cameron 1979). Tactics for resistance management include, among many others, the alternation, tank mixing, or use of prepackaged mixes containing active ingredients with different modes of action (Brent and Hollomon 2007; Ishii and Holloman 2015). Using site-specific materials such as FRAC group 3, group 7, or group 11 active ingredients with multisite materials such as FRAC group M5 are also encouraged.

The effective use of a fungicide program starting with chlorothalonil then switching to DMI fungicides (Pscheidt and Cluskey 2001) or the use of prepackaged combination materials (Pscheidt et al. 2017) for the management of EFB has been reported, but not the use of tank mixes. Tank mixes offer the grower flexibility in creating fungicide mixes with different modes of action at different rates or with active ingredients that may not be available in prepacked forms. Although each of the fungicides registered for EFB were effective alone, after 30 years of testing, there have been seasons where one or more of these FRAC groups were not among the most effective fungicides tested that year (J. W. Pscheidt, unpublished). Chlorothalonil, tested since 1989, with a FRAC group M5 multisite mode of action, has been among the most effective fungicides used each year (Pscheidt and Cluskey 2001; Pscheidt et al. 1990). Tank mixing chlorothalonil with other FRAC groups may help negate years when one of the active ingredients does not perform well against EFB. Copper hydroxide formulations (FRAC group M1) have resulted in inconsistent EFB management. EFB control with copper hydroxide was slightly less than chlorothalonil in some years and not significantly different than nontreated trees in other years, making it a poor choice as a mixing partner (Pscheidt and Cluskey 2001).

Although tank mixing may delay the development of fungicide resistance or manage EFB consistently over many years, there would be an increase in material costs. Despite record returns for producing 
hazelnut, ever cost-conscious growers are always looking for ways to reduce production inputs. Hazelnut growers frequently ask if each fungicide added to the tank mix should be at full or reduced rates. This question has been researched in many other pathosystems including apple scab, Septoria spp. on wheat, and various powdery mildews (Beckerman et al. 2015; Köller and Wilcox 1999; Metcalfe et al. 2000; Turechek and Köller 2004). The answer for resistance management is not simple and changes depending on host, pathogen, fungicides, and rates used.

This report summarizes the evaluation of tank mixes of DMI fungicides (FRAC group 3) or QoI fungicides (FRAC group 11) with chlorothalonil (FRAC group M5) at full or reduced rates for effectiveness against $A$. anomala.

\section{Materials and Methods}

In general, fungicide tank mix trials were conducted from 2006 to 2014 , where healthy hazelnut trees were planted each year in the dormant season, sprayed with test fungicides in the spring while exposed to ascospores of A. anomala, and then examined for EFB cankers in July the year after treatment due to the 2-year life cycle of this disease. Trees were used only once for a trial and then abandoned to serve as a source of inoculum for future trials.

Trials were conducted in one of two locations, including the North Willamette Research and Extension Center, Aurora, OR, during the 2006-07, 2007-08, and 2009-10 seasons and at the Botany and Plant Pathology Field Laboratory, Corvallis, OR during the 2010-11, 2011-12, 2013-14, and 2014-15 seasons. All trials were conducted in similar ways at each facility, with major exceptions as noted below.

Apparently healthy 2-year-old hazelnut trees were planted in either January or February into three rows (each up to $30 \mathrm{~m}$ long) at $0.6-\mathrm{m}$ spacing between both trees and rows. Trees were vegetatively propagated from the highly susceptible hazelnut cultivar Ennis and used in all trials. Prior to bud break, 300 to 400 cankered limbs from infected orchards were suspended directly above test trees on trellis-like supports. Weather was monitored using an Adcon meteorological station equipped with standard sensors. Ascospores were monitored each year using a rainwater ascospore trap (Pinkerton et al. 1998, 2001) placed among the test trees.

Fungicide treatments were arranged in a randomized complete block design each year. Each treatment consisted of four to eight single-tree replicates (four replicates in 2006-07; six replicates in 2007-08, 2009-10, and 2010-11; seven replicates in 2011-12; and eight replicates in 2013-14 and 2014-15). Fungicide suspensions were applied on two sides of the tree to runoff with a SoloPump-Style backpack sprayer equipped with a hand wand (Solo, Newport News, VA). In total, 3.0 liters was used per treatment from 2006-07 to 2007-08 and 0.9 liter was used from 2009-10 to 2014-15. Fungicide treatments were applied at bud break each year and again every 2 weeks (ranging from 12 to 15 days) for a total of four applications. Bud break occurred approximately mid-March each year, ranging from as early as 13 March 2014 to as late as 26 March 2009.

Most liquid chlorothalonil labels, such as Bravo Weather Stik (Syngenta Crop Protection LLC, Greensboro, NC), list a rate of 4.68 liters/ha (4 pt/acre) for EFB management. Assuming the use of water at 1,871 liters/ha (water at $200 \mathrm{gal} / \mathrm{acre}$ ), this translated to a rate or concentration of $946 \mathrm{ml}$ of fungicide per 378 liters of water (2 pt of fungicide per 100 gal of water). A "full rate" of Bravo Weather Stik, for use in these trials, was defined as $946 \mathrm{ml}$ of fungicide per 378 liters of water ( 2 pt of fungicide per 100 gal of water) and a "half-rate" was defined as $473 \mathrm{ml}$ of fungicide per 378 liters of water (1 pt of fungicide per $100 \mathrm{gal}$ of water). In general, then, all rates (concentrations) used for various fungicides in these trials were half ("full rate") to a quarter ("half-rate") of the labeled per area rate.

The following chlorothalonil (FRAC group M5) fungicides were evaluated, including Bravo Weather Stik at 473 or $946 \mathrm{ml}$ per 378 liters of water, Echo 720 (SipcamAgro USA, Inc., Durham, NC, 27713) at $473 \mathrm{ml}$ per 378 liters of water, Echo $90 \mathrm{DF}$ at 363 or $739 \mathrm{~g}$ per 378 liters of water, and Equus DF (Makhteshim Agan of
North America, Inc., ADAMA, Raleigh, NC) at 272 or 544 g per 378 liters of water. The following DMI (FRAC group 3) fungicides were evaluated, including metconazole as Quash 50 WDG (Valent U.S.A. Corporation, Walnut Creek CA) at 113 g per 378 liters of water, propiconazole as Orbit 3.6 EC or Tilt (Syngenta Crop Protection LLC) at 59 or $118 \mathrm{ml}$ per 378 liters of water, tebuconazole as Tebuzol 45 DF (United Phosphorus, Inc., King of Prussia, PA) at $113 \mathrm{~g}$ per 378 liters of water, and triflumizole as Procure 480 SC (MacDermid Agricultural Solutions, Inc. Waterbury, CT) at $118 \mathrm{ml}$ per 378 liters of water. The following QoI (FRAC group 11) fungicides were evaluated, including pyraclostrobin as Cabrio 20 EG (BASF Corporation, Research Triangle Park, NC) at 68 or 135 g per 378 liters of water and trifloxystrobin as Flint $50 \mathrm{WDG}$ at 14 or $28 \mathrm{~g}$ per 378 liters of water or Gem $500 \mathrm{SC}$ at $59 \mathrm{ml}$ per 378 liters of water (Bayer Crop Science LP, Research Triangle Park, NC).

A preliminary trial was initiated in 2006 with only four replications (Table 1) to determine whether there were any problems such as phytotoxicity or lack of disease control using mixes of a DMI and QoI fungicide together or with chlorothalonil. A more complete set of trials with six replications over three seasons (Table 2) evaluated various rates of propiconazole or pyraclostrobin with various rates of chlorothalonil. Additional DMI or QoI fungicides registered for EFB management were evaluated at various rates with chlorothalonil in 2011-12 (Table 3). In this trial, DMI fungicides were kept at full labeled rates while mixing with a half-rate of chlorothalonil. QoI fungicides were mixed at half labeled rates with a half-rate of chlorothalonil. Because tank mixing was not allowed with liquid formulations of chlorothalonil, dry formulations that do allow tank mixes were evaluated in 2013-14 and 2014-15 (Table 4).

During the application period each season, fungicide mixes were evaluated for physical tank compatibility while trees were evaluated for phytotoxicity or growth regulation activity. The first spring after planting, trees were painted with a 50\% solution of latex paint to prevent summer sunburn. Various herbicides such as glyphosate (Roundup; Monsanto Co., St. Louis), dichlobenil (Casoron 4G; MacDermid Agricultural Solutions, Inc.), or glufosinate (Rely; Bayer Crop Science LP) were used as needed each year to maintain a weed-free block of trees. Trees were fertilized with either agricultural grade urea (46-0-0) or a 16-16-16 complete fertilizer (Loveland Products, Loveland, CO) for moderate tree growth. Supplemental irrigation was also provided as needed during each growing season.

Due to the 2-year life cycle of this disease, the number of EFB cankers per tree was determined in July the year after fungicide application. Analysis of variance for each trial was based on $\log 10(x+1)$ transformation prior to mean comparison measures based on Fisher's protected least significant difference $(P=0.05)$ using Agricultural Research Manager software (Gylling Data Management, Inc., Brookings, SD). Canker means for individual trials are presented in tables below prior to transformation but letters are based on analysis of transformed data.

Cold injury to young trees in 2014 resulted in only $56 \%$ tree survival at the 2015 evaluation (data not presented). Due to limited tree survival, only treatments with at least four surviving trees were analyzed using a completely randomized design (Table 4).

\section{Results}

A sufficient number of ascospores as well as weather favorable for sporulation and infection occurred each spring, resulting in multiple cankers on small, highly susceptible trees (Pscheidt et al. 2017). Nontreated trees averaged from 3.0 to 9.8 EFB cankers per tree, depending on the season (Tables 1, 2, 3, and 4).

In the preliminary trial, trees treated with a combination of propiconazole and trifloxystrobin (Orbit and Flint) had a similar number of cankers as nontreated trees (Table 1). Trees treated with chlorothalonil (Bravo Weather Stik) had significantly fewer cankers than nontreated trees regardless of whether propiconazole or trifloxystrobin was in the tank mix. Trees treated with a half-rate of chlorothalonil mixed with a half-rate of either propiconazole or trifloxystrobin had a similar number of cankers as trees treated with a full rate of each material in the mix. 
All trees treated with various tank mixes of propiconazole or pyraclostrobin with chlorothalonil had significantly fewer cankers develop than on nontreated trees (Table 2). Few cankers developed on fungicide-treated trees during the 2007-08 season whereas treated trees generally averaged 2.1 and 1.1 cankers in 2009-10 and 2010-11, respectively. There were no significant differences in canker number among trees treated with various tank mixes of propiconazole or pyraclostrobin with chlorothalonil. Trees treated with a tank mix of propiconazole with pyraclostrobin at full rates had significantly fewer cankers than nontreated trees. In 2007-08, trees treated with a tank mix of propiconazole with pyraclostrobin at full rates had significantly more cankers than other tank mixes,

Table 1. Number of eastern filbert blight cankers on trees treated with a fungicide mixture of propiconazole (FRAC group 3) or trifloxystrobin (FRAC group 11) with chlorothalonil (FRAC group M5) during the 2006-07 season ${ }^{\mathrm{x}}$

\begin{tabular}{|c|c|c|c|c|}
\hline Active ingredients & FRAC group & $\begin{array}{l}\text { Formulated product } \\
\text { (trade name) }\end{array}$ & $\begin{array}{l}\text { Formulated product per } \\
378 \text { liters of water }\end{array}$ & $\begin{array}{l}\text { Average number of } \\
\text { cankers/tree }^{\mathrm{z}}\end{array}$ \\
\hline Nontreated & $\ldots$ & $\ldots$ & $\ldots$ & $5.3 \mathrm{a}$ \\
\hline $\begin{array}{l}\text { Propiconazole + } \\
\text { chlorothalonil }\end{array}$ & $\begin{array}{l}3 \\
\text { M5 }\end{array}$ & $\begin{array}{l}\text { Orbit 3.6 EC } \\
\text { Bravo Weather Stik }\end{array}$ & $\begin{array}{r}74 \mathrm{ml} \\
946 \mathrm{ml}\end{array}$ & $1.3 \mathrm{bc}$ \\
\hline $\begin{array}{l}\text { Propiconazole }+ \\
\text { chlorothalonil }\end{array}$ & $\begin{array}{l}3 \\
\text { M5 }\end{array}$ & $\begin{array}{l}\text { Orbit 3.6 EC } \\
\text { Bravo Weather Stik }\end{array}$ & $\begin{array}{r}37 \mathrm{ml} \\
473 \mathrm{ml}\end{array}$ & $0.5 \mathrm{c}$ \\
\hline $\begin{array}{l}\text { Trifloxystrobin + } \\
\text { chlorothalonil }\end{array}$ & $\begin{array}{l}11 \\
\text { M5 }\end{array}$ & $\begin{array}{l}\text { Flint } 50 \text { WG } \\
\text { Bravo Weather Stik }\end{array}$ & $\begin{array}{c}28 \mathrm{~g} \\
946 \mathrm{ml}\end{array}$ & $1.0 \mathrm{bc}$ \\
\hline $\begin{array}{l}\text { Trifloxystrobin + } \\
\text { chlorothalonil }\end{array}$ & $\begin{array}{l}11 \\
\text { M5 }\end{array}$ & $\begin{array}{l}\text { Flint } 50 \text { WG } \\
\text { Bravo Weather Stik }\end{array}$ & $\begin{array}{c}14 \mathrm{~g} \\
473 \mathrm{ml}\end{array}$ & $0.8 \mathrm{c}$ \\
\hline $\begin{array}{c}\text { Propiconazole }+ \\
\text { trifloxystrobin }\end{array}$ & $\begin{array}{l}3 \\
11\end{array}$ & $\begin{array}{l}\text { Orbit } 3.6 \mathrm{EC} \\
\text { Flint } 50 \mathrm{WG}\end{array}$ & $\begin{array}{l}74 \mathrm{ml} \\
28 \mathrm{~g}\end{array}$ & $2.8 \mathrm{ab}$ \\
\hline $\begin{array}{c}\text { Propiconazole }+ \\
\text { trifloxystrobin }\end{array}$ & $\begin{array}{l}3 \\
11\end{array}$ & $\begin{array}{l}\text { Orbit } 3.6 \mathrm{EC} \\
\text { Flint } 50 \mathrm{WG}\end{array}$ & $\begin{array}{l}37 \mathrm{ml} \\
14 \mathrm{~g}\end{array}$ & $5.5 \mathrm{a}$ \\
\hline
\end{tabular}

${ }^{\mathrm{x}}$ Fungicides were applied in the spring of 2006 beginning at bud break and continuing at 2-week intervals for a total of four applications. The number of eastern filbert blight cankers per tree was determined the summer after fungicide application in 2007.

y Numbers in bold indicate rates at half commercial labeled recommendation.

${ }^{\mathrm{z}}$ Average number of cankers per tree was calculated prior to analysis. Analysis of variance was based on $\log 10(x+1)$ transformation. Means followed by the same letter did not differ based on Fisher's protected least significant difference (trial $P$ value $=0.0006$ ).

Table 2. Number of eastern filbert blight cankers on trees treated with a fungicide mixture of propiconazole (FRAC group 3) or pyraclostrobin (FRAC group 11) with chlorothalonil (FRAC group M5) during the 2007-08, 2009-10, and 2010-11 seasonsw

\begin{tabular}{|c|c|c|c|c|c|c|}
\hline \multirow[b]{2}{*}{ Active ingredients } & \multirow[b]{2}{*}{ FRAC group } & \multicolumn{2}{|c|}{ Formulated product } & \multicolumn{3}{|c|}{ Average number of cankers/tree ${ }^{x}$} \\
\hline & & Trade name & Per 378 liters of water ${ }^{y}$ & $2007-08^{z}$ & 2009-10 & 2010-11 \\
\hline Nontreated & $\ldots$ & $\ldots$ & $\ldots$ & $4.5 \mathrm{a}$ & $9.8 \mathrm{a}$ & $3.9 \mathrm{a}$ \\
\hline $\begin{array}{c}\text { Propiconazole }+ \\
\text { chlorothalonil }\end{array}$ & $\begin{array}{l}3 \\
\text { M5 }\end{array}$ & $\begin{array}{l}\text { Orbit 3.6 EC } \\
\text { Bravo Weather Stik }\end{array}$ & $\begin{array}{l}118 \mathrm{ml} \\
946 \mathrm{ml}\end{array}$ & $0.0 \mathrm{c}$ & $2.5 \mathrm{~b}$ & $0.7 \mathrm{~b}$ \\
\hline $\begin{array}{l}\text { Propiconazole + } \\
\text { chlorothalonil }\end{array}$ & $\begin{array}{l}3 \\
\text { M5 }\end{array}$ & $\begin{array}{l}\text { Orbit 3.6 EC } \\
\text { Bravo Weather Stik }\end{array}$ & $\begin{array}{r}\mathbf{5 9} \mathbf{~ m l} \\
946 \mathrm{ml}\end{array}$ & $0.0 \mathrm{c}$ & $1.8 \mathrm{~b}$ & $1.0 \mathrm{~b}$ \\
\hline $\begin{array}{l}\text { Propiconazole }+ \\
\text { chlorothalonil }\end{array}$ & $\begin{array}{l}3 \\
\text { M5 }\end{array}$ & $\begin{array}{l}\text { Orbit 3.6 EC } \\
\text { Bravo Weather Stik }\end{array}$ & $\begin{array}{l}118 \mathrm{ml} \\
\mathbf{4 7 3} \mathrm{ml}\end{array}$ & $0.0 \mathrm{c}$ & $2.0 \mathrm{~b}$ & $0.5 \mathrm{~b}$ \\
\hline $\begin{array}{l}\text { Propiconazole + } \\
\text { chlorothalonil }\end{array}$ & $\begin{array}{l}3 \\
\text { M5 }\end{array}$ & $\begin{array}{l}\text { Orbit } 3.6 \text { EC } \\
\text { Bravo Weather Stik }\end{array}$ & $\begin{array}{r}59 \mathrm{ml} \\
473 \mathrm{ml}\end{array}$ & $0.3 \mathrm{bc}$ & $1.3 \mathrm{~b}$ & $1.5 \mathrm{~b}$ \\
\hline $\begin{array}{l}\text { Pyraclostrobin + } \\
\text { chlorothalonil }\end{array}$ & $\begin{array}{l}11 \\
\text { M5 }\end{array}$ & $\begin{array}{l}\text { Cabrio } 20 \text { EG } \\
\text { Bravo Weather Stik }\end{array}$ & $\begin{array}{l}135 \mathrm{~g} \\
946 \mathrm{ml}\end{array}$ & $0.0 \mathrm{c}$ & $1.2 \mathrm{~b}$ & $0.8 \mathrm{~b}$ \\
\hline $\begin{array}{l}\text { Pyraclostrobin + } \\
\text { chlorothalonil }\end{array}$ & $\begin{array}{l}11 \\
\text { M5 }\end{array}$ & $\begin{array}{l}\text { Cabrio } 20 \text { EG } \\
\text { Bravo Weather Stik }\end{array}$ & $\begin{array}{c}\mathbf{6 8} \mathbf{g} \\
946 \mathrm{ml}\end{array}$ & $0.0 \mathrm{c}$ & $1.7 \mathrm{~b}$ & $1.2 \mathrm{~b}$ \\
\hline $\begin{array}{l}\text { Pyraclostrobin + } \\
\text { chlorothalonil }\end{array}$ & $\begin{array}{l}11 \\
\text { M5 }\end{array}$ & $\begin{array}{l}\text { Cabrio } 20 \text { EG } \\
\text { Bravo Weather Stik }\end{array}$ & $\begin{array}{l}135 \mathrm{~g} \\
\mathbf{4 7 3} \mathbf{~ m l}\end{array}$ & $0.0 \mathrm{c}$ & $1.7 \mathrm{~b}$ & $1.2 \mathrm{~b}$ \\
\hline $\begin{array}{l}\text { Pyraclostrobin }+ \\
\text { chlorothalonil }\end{array}$ & $\begin{array}{l}11 \\
\text { M5 }\end{array}$ & $\begin{array}{l}\text { Cabrio } 20 \text { EG } \\
\text { Bravo Weather Stik }\end{array}$ & $\begin{array}{c}68 \mathrm{~g} \\
473 \mathrm{ml}\end{array}$ & $0.0 \mathrm{c}$ & $3.3 \mathrm{~b}$ & $1.5 \mathrm{~b}$ \\
\hline $\begin{array}{c}\text { Propiconazole }+ \\
\text { pyraclostrobin }\end{array}$ & $\begin{array}{l}3 \\
11\end{array}$ & $\begin{array}{l}\text { Orbit } 3.6 \mathrm{EC} \\
\text { Cabrio } 20 \mathrm{EG}\end{array}$ & $\begin{array}{l}118 \mathrm{ml} \\
135 \mathrm{~g}\end{array}$ & $0.5 \mathrm{~b}$ & $3.2 \mathrm{~b}$ & $1.7 \mathrm{~b}$ \\
\hline Trial $P$ value & $\ldots$ & $\ldots$ & $\ldots$ & 0.0001 & 0.0001 & 0.006 \\
\hline
\end{tabular}


except when both propiconazole and chlorothalonil were at halfrates.

All trees treated with tank mixes of a DMI (FRAC group 3) or QoI (FRAC group 11) fungicide registered for EFB management with half-rates of chlorothalonil had significantly fewer cankers than nontreated trees (Table 3). There were no significant differences in canker number among trees treated with various DMI fungicides mixed with a half-rate of chlorothalonil. There were also no significant differences in canker number among trees treated with various QoI fungicides mixed with chlorothalonil with each at half the labeled rate.

All fungicide-treated trees had significantly fewer cankers develop than on nontreated trees in the 2013-14 and 2014-15 seasons (Table 4). In 2013-14, trees treated with the full rate of either

Table 3. Number of eastern filbert blight cankers on trees treated with a fungicide mixture of demethylation (DMI, FRAC group 3) or quinone outside inhibitor (QoI, FRAC group 11) fungicides with chlorothalonil (FRAC group M5) during the 2011-12 season ${ }^{\mathrm{x}}$

\begin{tabular}{|c|c|c|c|c|}
\hline Active ingredients & FRAC group & $\begin{array}{l}\text { Formulated product } \\
\text { (trade name) }\end{array}$ & $\begin{array}{l}\text { Formulated product per } \\
378 \text { liters of water }\end{array}$ & $\begin{array}{l}\text { Average number of } \\
\text { cankers/tree }\end{array}$ \\
\hline Nontreated & $\ldots$ & $\ldots$ & $\ldots$ & $6.6 \mathrm{a}$ \\
\hline $\begin{array}{l}\text { Propiconazole }+ \\
\text { chlorothalonil }\end{array}$ & $\begin{array}{l}3 \\
\text { M5 }\end{array}$ & $\begin{array}{l}\text { Tilt 3.6 EC } \\
\text { Bravo Weather Stik }\end{array}$ & $\begin{array}{l}118 \mathrm{ml} \\
946 \mathrm{ml}\end{array}$ & $3.1 \mathrm{~b}$ \\
\hline $\begin{array}{c}\text { Propiconazole }+ \\
\text { chlorothalonil }\end{array}$ & $\begin{array}{l}3 \\
\text { M5 }\end{array}$ & $\begin{array}{l}\text { Tilt 3.6 EC } \\
\text { Bravo Weather Stik }\end{array}$ & $\begin{array}{l}118 \mathrm{ml} \\
\mathbf{4 7 3} \mathrm{ml}\end{array}$ & $3.6 \mathrm{~b}$ \\
\hline $\begin{array}{l}\text { Triflumizole }+ \\
\text { chlorothalonil }\end{array}$ & $\begin{array}{l}3 \\
\text { M5 }\end{array}$ & $\begin{array}{l}\text { Procure } 480 \text { SC } \\
\text { Bravo Weather Stik }\end{array}$ & $\begin{array}{l}118 \mathrm{ml} \\
\mathbf{4 7 3} \mathrm{ml}\end{array}$ & $2.6 \mathrm{~b}$ \\
\hline $\begin{array}{r}\text { Tebuconazole }+ \\
\text { chlorothalonil }\end{array}$ & $\begin{array}{l}3 \\
\text { M5 }\end{array}$ & $\begin{array}{l}\text { Tebuzol } 45 \text { DF } \\
\text { Bravo Weather Stik }\end{array}$ & $\begin{array}{l}113 \mathrm{~g} \\
\mathbf{4 7 3} \mathrm{ml}\end{array}$ & $2.1 \mathrm{~b}$ \\
\hline $\begin{array}{l}\text { Metconazole + } \\
\text { chlorothalonil }\end{array}$ & $\begin{array}{l}3 \\
\text { M5 }\end{array}$ & $\begin{array}{l}\text { Quash } 50 \text { WDG } \\
\text { Bravo Weather Stik }\end{array}$ & $\begin{array}{l}113 \mathrm{~g} \\
\mathbf{4 7 3} \mathrm{ml}\end{array}$ & $2.3 \mathrm{~b}$ \\
\hline $\begin{array}{l}\text { Pyraclostrobin + } \\
\text { chlorothalonil }\end{array}$ & $\begin{array}{l}11 \\
\text { M5 }\end{array}$ & $\begin{array}{l}\text { Cabrio } 20 \text { EG } \\
\text { Bravo Weather Stik }\end{array}$ & $\begin{array}{l}135 \mathrm{~g} \\
946 \mathrm{ml}\end{array}$ & $1.6 \mathrm{~b}$ \\
\hline $\begin{array}{l}\text { Pyraclostrobin }+ \\
\text { chlorothalonil }\end{array}$ & $\begin{array}{l}11 \\
\text { M5 }\end{array}$ & $\begin{array}{l}\text { Cabrio } 20 \text { EG } \\
\text { Bravo Weather Stik }\end{array}$ & $\begin{array}{c}68 \mathrm{~g} \\
473 \mathrm{ml}\end{array}$ & $2.1 \mathrm{~b}$ \\
\hline $\begin{array}{l}\text { Trifloxystrobin + } \\
\text { chlorothalonil }\end{array}$ & $\begin{array}{l}11 \\
\text { M5 }\end{array}$ & $\begin{array}{l}\text { Gem } 500 \text { SC } \\
\text { Bravo Weather Stik }\end{array}$ & $\begin{array}{r}59 \mathrm{ml} \\
473 \mathrm{ml}\end{array}$ & $2.7 \mathrm{~b}$ \\
\hline
\end{tabular}

${ }^{\mathrm{x}}$ Fungicides were applied in the spring of 2011 beginning at bud break and continuing at 2-week intervals for a total of four applications. The number of eastern filbert blight cankers per tree was determined the summer after fungicide application in 2012.

y Numbers in bold indicate rates at half commercial labeled recommendation.

${ }^{\mathrm{z}}$ Average number of cankers per tree was calculated prior to analysis. Analysis of variance was based on $\log 10(x+1)$ transformation. Means followed by the same letter did not differ based on Fisher's protected least significant difference (trial $P$ value $=0.0167$ ).

Table 4. Number of eastern filbert blight cankers on trees treated alone or with a mixture of pyraclostrobin (FRAC group 11) and chlorothalonil (FRAC group M5) during the 2013-14 and 2014-15 seasons ${ }^{\mathrm{w}}$

\begin{tabular}{|c|c|c|c|c|c|}
\hline \multirow[b]{2}{*}{ Active ingredients } & \multirow[b]{2}{*}{ FRAC group } & \multicolumn{2}{|c|}{ Formulated product ${ }^{\mathbf{x}}$} & \multicolumn{2}{|c|}{$\begin{array}{l}\text { Average number of } \\
\text { cankers/tree }^{y}\end{array}$} \\
\hline & & Trade name & Per 378 liters of water & $2013-14^{z}$ & 2014-15 \\
\hline Nontreated & $\ldots$ & $\ldots$ & $\ldots$ & $4.6 \mathrm{a}$ & $3.0 \mathrm{a}$ \\
\hline Pyraclostrobin & 11 & Cabrio 20 EG & $135 \mathrm{~g}$ & $0.3 \mathrm{~cd}$ & - \\
\hline Pyraclostrobin & 11 & Cabrio 20 EG & $68 \mathrm{~g}$ & $1.5 \mathrm{~b}$ & - \\
\hline \multirow{2}{*}{$\begin{array}{l}\text { Pyraclostrobin + } \\
\text { chlorothalonil }\end{array}$} & 11 & Cabrio 20 EG & $68 \mathrm{~g}$ & & \\
\hline & M5 & Echo 720 & $473 \mathrm{ml}$ & $1.1 \mathrm{bcd}$ & $0.3 \mathrm{~b}$ \\
\hline \multirow{2}{*}{$\begin{array}{l}\text { Pyraclostrobin + } \\
\text { chlorothalonil }\end{array}$} & 11 & Cabrio 20 EG & $68 \mathrm{~g}$ & & \\
\hline & M5 & Echo 90 DF & $363 \mathrm{~g}$ & $0.5 \mathrm{~cd}$ & - \\
\hline Chlorothalonil & M5 & Echo 90 DF & $363 \mathrm{~g}$ & $0.9 \mathrm{bc}$ & - \\
\hline Chlorothalonil & M5 & Echo $90 \mathrm{DF}$ & $739 \mathrm{~g}$ & $0.3 \mathrm{~d}$ & - \\
\hline \multirow{2}{*}{$\begin{array}{l}\text { Pyraclostrobin + } \\
\text { chlorothalonil }\end{array}$} & 11 & Cabrio 20 EG & $68 \mathrm{~g}$ & & \\
\hline & M5 & Equus DF & $272 \mathrm{~g}$ & - & $0.5 \mathrm{~b}$ \\
\hline Chlorothalonil & M5 & Equus DF & $272 \mathrm{~g}$ & - & $0.5 \mathrm{~b}$ \\
\hline Chlorothalonil & M5 & Equus DF & $544 \mathrm{~g}$ & - & $0.0 \mathrm{~b}$ \\
\hline Trial $P$ value & $\ldots$ & $\ldots$ & $\ldots$ & 0.0001 & 0.0002 \\
\hline \multicolumn{6}{|c|}{$\begin{array}{l}\text { w Fungicides were applied in the spring beginning at bud break and continuing at 2-week intervals for a total of four applications. The number of eastern filbert } \\
\text { blight cankers per tree was determined the summer after fungicide application. }\end{array}$} \\
\hline \multicolumn{6}{|c|}{$x$ Numbers in bold indicate rates at half commercial labeled recommendation. } \\
\hline \multirow{3}{*}{\multicolumn{6}{|c|}{$\begin{array}{l}\text { y Average number of cankers per tree was calculated prior to analysis. Analysis of variance was based on } \log 10(x+1) \text { transformation. A complete randomized } \\
\text { block design with eight replications was used in } 2013-14 \text { but, due to cold injury, only treatments in } 2014-15 \text { with at least four surviving trees were analyzed } \\
\text { using a completely randomized design. Means followed by the same letter did not differ based on Fisher's protected least significant difference }(P=0.05) \text {; } \\
\text { - indicates treatment not conducted that year. }\end{array}$}} \\
\hline & & & & & \\
\hline & & & & & \\
\hline $\mathrm{z}$ The first year listec & fungicides were a & e spring and the & listed was when the numbe & was determi & the 2-year \\
\hline
\end{tabular}


chlorothalonil (as Echo 90 DF) or pyraclostrobin alone or the halfrate each of chlorothalonil (as Echo $90 \mathrm{DF}$ ) plus pyraclostrobin had significantly fewer cankers than trees treated with the half-rate of pyraclostrobin alone. Trees treated with the full rate of chlorothalonil (as Echo 90 DF) alone had significantly fewer cankers than trees treated with the half-rate of chlorothalonil (as Echo 90 DF) alone. In 2014-15, trees treated with the full rate of chlorothalonil (as Equus DF) did not develop cankers; however, the number of cankers on trees treated with the half-rate of chlorothalonil (as Equus DF) alone or tank mixed with a half-rate of pyraclostrobin were not significantly different. There were no significant differences in canker number among trees treated with pyraclostrobin mixed with either liquid or dry formulations of chlorothalonil.

No physical tank incompatibility such as particle flocking or precipitation was observed with any fungicide mix. Only trees treated with a mix of metconazole and chlorothalonil in 2011-12 had any acute phytotoxicity reactions. These trees developed a marginal leaf necrosis after several applications. All trees treated with propiconazole had darker green and smaller leaves than nontreated trees (evaluation data not presented). Trees grew out of this plant growth regulation effect several weeks after the last application.

\section{Discussion}

The use of chlorothalonil (FRAC group M5) in a mix with a DMI (FRAC group 3) or QoI (FRAC group 11) fungicide was an effective treatment for EFB. There were no significant differences in canker number among the various mixes used with chlorothalonil each year, even if each component of the mix was at half the labeled rate (Tables $1,2,3$, and 4). Different liquid or dry formulations of chlorothalonil were equally effective in a tank mix for EFB control. Previous research associated reduced rates of chlorothalonil (used alone) with inconsistent EFB control (Pscheidt and Cluskey 2001), which was also evident in this study (Table 4). The half-rate of pyraclostrobin alone, tested only once, resulted in more cankers that the full rate (Table 4), indicating that this reduced rate should either be avoided or used in a tank mix with another fungicide.

The combination of propiconazole (Orbit, FRAC group 3) tank mixed with trifloxystrobin (Flint, FRAC group 11) was not effective (Table 1) whereas propiconazole tank mixed with pyraclostrobin (FRAC group 11) resulted in significantly fewer EFB cankers compared with nontreated trees (Table 2). Rainfall and temperatures between seasons were similar and would not explain the difference (Pscheidt et al. 2017). There may have been compatibility problems mixing Orbit with Flint because the prepackaged combination known as Stratego (propiconazole + trifloxystrobin) resulted in good EFB control (Pscheidt et al. 2017) and is registered for hazelnut (Pscheidt and Ocamb 2017). Obvious physical compatibility problems in the tank when these two materials were mixed were not observed. At this time, there is no commercial prepackaged formulation of propiconazole plus pyraclostrobin registered for hazelnut.
The advantage in EFB control of using chlorothalonil in a mix may be weather dependent. The use of chlorothalonil in a mix with a DMI or QoI fungicide resulted in fewer cankers than when a DMI was mixed with a QoI in 2006-07 and 2007-08 but not in 2009-10 or 2010-11 (Tables 1 and 2). Residual fungicide activity, especially for nonsystemic contact materials such as chlorothalonil, can be reduced by high temperatures (that accelerate shoot growth that outgrows chemical protection) or high rainfall between applications (Fife and Nokes 2002). Mean daily temperatures during the 8-week infection period did not explain the difference because the 2006-07, 2007-08, and 2009-10 seasons were similar $\left(10.8,10.4\right.$, and $10.8^{\circ} \mathrm{C}$, respectively) but slightly cooler for $2010-11\left(8.8^{\circ} \mathrm{C}\right)$. Rainfall during the 8-week infection period might explain the difference, in part, because the 2006-07 and 2007-08 seasons had less rain (11.7 and $12 \mathrm{~cm}$, respectively) than the 2009-10 and 2010-11 seasons (16.1 and $23.6 \mathrm{~cm}$, respectively). Tank mixing chlorothalonil with other FRAC groups may result in better EFB control in years where one of the active ingredients does not perform well against EFB. At this time, there are no commercial prepackaged formulations of chlorothalonil with other fungicides registered for hazelnut.

Label instructions discourage the continuous consecutive use of fungicides that are at risk of selecting resistant pathogens. Tank mixing multisite materials such as chlorothalonil along with the rotation of site-specific materials such as FRAC group 3, group 7, or group 11 active ingredients is an accepted tactic, among many others, for resistance management (Brent and Hollomon 2007; Ishii and Holloman 2015). Hazelnut growers can safely use custom mixes of chlorothalonil with several DMI or QoI fungicides and maintain adequate EFB control. Custom mixes with metconazole products such as Quash 50 WDG (DMI, FRAC group 3) should be avoided due to phytotoxicity. Mixes with the formulated products Orbit (propiconazole, FRAC group 3) and Flint (trifloxystrobin, FRAC group 11) should also be avoided due to poor EFB control.

Although resistance to fungicides has not been found or suspected, based on other pathosystems (Beckerman et al. 2015; Ishii and Holloman 2015), we would hypothesize that $A$. anomala might develop polygenic resistance mechanisms toward DMI fungicides such as propiconazole but monogenic resistance to QoI such as pyraclostrobin. Use of reduced rates of DMI is discouraged for resistance management (Brent and Hollomon 2007) and, thus, full rates should be maintained when used in a tank mix. The recommendation for resistance management of QoI focuses on tank mixes (as well as rotations and minimal use) but rates were not addressed (Brent and Hollomon 2007). It is suspected that use of reduced rates of a QoI would not necessarily encourage resistance development beyond using the group in the first place and, thus, reduced rates could be used in a tank mix.

When using tank mixes for EFB and resistance management, DMI should remain at full rates while mixing with a half-rate of chlorothalonil. In contrast, QoI and chlorothalonil could both be used at halfrates and still maintain acceptable EFB control.

Table 5. Fungicide application costs when used alone or in a tank mix

\begin{tabular}{|c|c|c|c|c|c|c|}
\hline \multirow[b]{2}{*}{ Active ingredients } & \multirow[b]{2}{*}{ FRAC group } & \multicolumn{2}{|c|}{ Formulated product } & \multicolumn{3}{|c|}{ Cost/ha $(\$)^{\mathbf{w}}$} \\
\hline & & Trade name ${ }^{x}$ & Per hectare $^{y}$ & Fungicide & Machine and labor & Total $^{\mathbf{z}}$ \\
\hline Chlorothalonil & M5 & Bravo Weather Stik & 4.68 liters & 40.48 & 31.43 & 71.91 \\
\hline Chlorothalonil & M5 & Bravo Weather Stik & 2.34 liters & 20.24 & 31.43 & 51.67 \\
\hline Propiconazole & 3 & Orbit/Tilt 3.6 EC & $585 \mathrm{ml}$ & 16.27 & 31.43 & 47.70 \\
\hline Propiconazole & 3 & Orbit/Tilt 3.6 EC & $292 \mathrm{ml}$ & 8.14 & 31.43 & 39.57 \\
\hline Pyraclostrobin & 11 & Cabrio 20 EG & $665 \mathrm{~g}$ & 53.47 & 31.43 & 84.90 \\
\hline Pyraclostrobin & 11 & Cabrio 20 EG & $333 \mathrm{~g}$ & 26.74 & 31.43 & 58.17 \\
\hline $\begin{array}{c}\text { Propiconazole + } \\
\text { chlorothalonil }\end{array}$ & $\begin{array}{l}3 \\
\text { M5 }\end{array}$ & $\begin{array}{l}\text { Orbit 3.6 EC } \\
\text { Bravo Weather Stik }\end{array}$ & $\begin{array}{l}585 \mathrm{ml} \\
2.34 \text { liters }\end{array}$ & $\begin{array}{l}16.27 \\
20.24\end{array}$ & 31.43 & 67.94 \\
\hline $\begin{array}{l}\text { Pyraclostrobin + } \\
\text { chlorothalonil }\end{array}$ & $\begin{array}{l}11 \\
\text { M5 }\end{array}$ & $\begin{array}{l}\text { Cabrio } 20 \text { EG } \\
\text { Bravo Weather Stik }\end{array}$ & $\begin{array}{l}333 \mathrm{~g} \\
2.34 \text { liters }\end{array}$ & $\begin{array}{l}26.74 \\
20.24\end{array}$ & 31.43 & 78.41 \\
\hline
\end{tabular}

\footnotetext{
${ }^{\mathrm{w}}$ Costs are based on averages, as outlined by Miller et al. (2013).

${ }^{x}$ Orbit is no longer available but the comparable formulation Tilt is registered for hazelnut at similar prices.

y Based on current label rates.

${ }^{\mathrm{z}}$ Total cost $=$ fungicide cost + machine and labor costs.
} 
Fungicide labels generally express rates on a per-area basis, which can be hard to translate to small-plot experimental trials. The thorough coverage obtained using a backpack sprayer on a small, 2-year-old tree is similar to the coverage obtained in a mature, full-canopy orchard when using water at 1,871 liters/ha ( $200 \mathrm{gal} / \mathrm{acre})$ or more. The "full-rate" concentration of chlorothalonil used in this research ( $2 \mathrm{pt}$ of fungicide per 100 gal of water) has been shown to be effective for EFB management in small-plot trials for many years (Pscheidt and Cluskey 2001).

Although tank mixing at full rates would substantially increase material costs, use of half-rates would keep costs lower or even reduce costs (Table 5). Fungicide application costs for chlorothalonil or pyraclostrobin include product costs of $\$ 40.48$ or $53.47 / \mathrm{ha}(\$ 16.38$ or 21.64/acre) (Bravo at 4.68 liters/ha or Cabrio at $666 \mathrm{~g} / \mathrm{ha}$ ) plus variable machine and labor costs of $\$ 31.43 /$ application/ha $(\$ 12.72 /$ application/acre), for a total cost of \$71.91 and \$84.90/application/ha (\$29.10 and \$34.36/application/acre), respectively (Miller et al. 2013). The label for propiconazole (Orbit or Tilt) has a range of rates, with the highest at $585 \mathrm{ml} / \mathrm{ha}(8 \mathrm{fl} \mathrm{oz} / \mathrm{acre})$, which translated into a cost of $\$ 47.70 /$ application/ha (\$19.30/application/acre) using the same machine and labor costs (Miller et al. 2013). Using the rates proposed in the previous paragraph, tank mixing a full rate of Tilt with a halfrate of Bravo results in an application cost of $\$ 16.27+(\$ 40.48 / 2)+$ $\$ 31.43=\$ 67.94 /$ application $/$ ha $(\$ 6.58+[\$ 16.38 / 2]+\$ 12.72=$ $\$ 27.49 /$ application/acre). Even if a half-rate of Tilt were useful, it would only be a savings of \$8.14/application/ha (\$3.29/application/ acre). Tank mixing a half-rate each of Cabrio and Bravo, however, results in an application cost of $(\$ 53.47 / 2)+(\$ 40.48 / 2)+\$ 31.43=$ $\$ 78.41 /$ application $/$ ha $([\$ 21.64 / 2]+[\$ 16.38 / 2]+\$ 12.72=$ $\$ 31.73 /$ application/acre) which is lower than using Cabrio alone at the full rate. Actual costs will vary depending on year, supplier, volume, and the grower's own resources.

Fungicide management of EFB will include proper timing, application technique for good coverage, rotation of products with one or more different modes of action, as well as tank mixes. In the near future, tank mixing chlorothalonil with at-risk fungicides can help maintain consistent EFB control and should help prevent or delay the emergence of fungicide-resistant $A$. anomala isolates. In the long term, future fungicide use will decline as the hazelnut industry in the Pacific Northwest (PNW) converts from EFB-susceptible to EFBresistant cultivars (Muehlbauer at al. 2014). This expensive transition will take many years as old orchards become unproductive from EFB. Expensive new resistant cultivars such as Jefferson will still need fungicide applications for the first year or two after transplanting (Pscheidt and Ocamb 2017). These new orchards are being planted in high-inoculum situations such as within or downwind of heavily infected orchards, necessitating the need for continued fungicide use. Fungicides would also be necessary if isolates of $A$. anomala develop or are brought into the PNW that can overcome the single dominant gene used in these resistant cultivars.

\section{Literature Cited}

Beckerman, J. L., Sundin, G. W., and Rosenberger, D. A. 2015. Do some IPM concepts contribute to the development of fungicide resistance? Lessons learned from the apple scab pathosystem in the United States. Pest Manage. Sci. 71:331-342.

Brent, K. J., and Hollomon, D. W. 2007. Fungicide Resistance in Crop Pathogens: How Can It Be Managed? FRAC Monograph 1. 2nd, revised ed. Fungicide Resistance Action Committee, Brussels, Belgium.

Fife, J. P., and Nokes, S. E. 2002. Evaluation of the effect of rainfall intensity and duration on the persistence of chlorothalonil on processing tomato foliage. Crop Prot. 21:733-740.

Gottwald, T. R., and Cameron, H. R. 1979. Studies in the morphology and life history of Anisogramma anomala. Mycologia 71:1107-1126.

Heckert, S., Pscheidt, J. W., and Cluskey, S. A. 2014. Disease incidence and ascospore dispersal from cut hazelnut branches colonized by Anisogramma anomala. Plant Dis. 98:834-838.

Ishii, H., and Holloman, D. W., eds. 2015. Fungicide Resistance in Plant Pathogens. Springer, Tokyo.

Johnson, K. B., Mehlenbacher, S. A., Stone, J. K., Pscheidt, J. W., and Pinkerton, J. N. 1996. Eastern filbert blight of European hazelnuts: It's becoming a manageable disease. Plant Dis. 80:1308-1316.

Johnson, K. B., Pscheidt, J. W., and Pinkerton, J. N. 1993. Evaluation of chlorothalonil, fenarimol, and flusilazole for control of eastern filbert blight. Plant Dis. 77:831-837.

Köller, W., and Wilcox, W. F. 1999. Evaluation of tactics for managing resistance of Venturia inaequalis to sterol demethylation inhibitors. Plant Dis. 83:857-863.

Metcalfe, R. J., Shaw, M. W., and Russell, P. E. 2000. The effect of dose and mobility on the strength of selection for DMI fungicide resistance in inoculated field experiments. Plant Pathol. 49:546-557.

Miller, M., Seavert, C. F., and Olsen, J. L. 2013. Orchard economics: The costs and returns of establishing and producing hazelnuts in the Willamette Valley. Oregon State University Extension Service, Corvallis, OR. http://arec. oregonstate.edu/oaeb/files/pdf/AEB0043.pdf

Muehlbauer, M. F., Honig, J. A., Capik, J. M., Vaiciunas, J. N., and Molnar, T. J. 2014. Characterization of eastern filbert blight-resistant hazelnut germplasm using microsatellite markers. J. Am. Soc. Hortic. Sci. 139:399-432.

Oregon Department of Agriculture. 2015. Page 52 in: Oregon Agripedia. Online publication. Oregon Department of Agriculture, Salem. http://www.oregon. gov/ODA/shared/Documents/Publications/Administration/Agripedia.pdf

Pinkerton, J. N., Johnson, K. B., Aylor, D. E., and Stone, J. K. 2001. Spatial and temporal increase of eastern filbert blight in European hazelnut orchards in the Pacific Northwest. Phytopathology 91:1214-1223.

Pinkerton, J. N., Johnson, K. B., Stone, J. K., and Ivors, K. L. 1998. Maturation and seasonal discharge pattern of ascospores of Anisogramma anomala. Phytopathology 88:1165-1173.

Pscheidt, J. W., and Cluskey, S. 2001. Fungicide control of eastern filbert blight. Acta Hortic. 556:411-418.

Pscheidt, J. W., Heckert, S., and Cluskey, S. A. 2017. Evaluation of quinone outside and succinate dehydrogenase inhibitors for effectiveness against eastern filbert blight of hazelnut. Plant Dis. 101:1868-1873.

Pscheidt, J. W., Johnson, K. B., and Cameron, H. R. 1990. Comparison of fungicides for control of eastern filbert blight, 1989 season. Fungic. Nematicide Tests 45:86.

Pscheidt, J. W., and Ocamb, C. M., eds. 2017. Pacific Northwest Plant Disease Management Handbook. Oregon State University, Corvallis.

Stone, J. K., Johnson, K. B., Pinkerton, J. N., and Pscheidt, J. W. 1992. Natura infection period and susceptibility of vegetative seedlings of European hazelnut to Anisogramma anomala. Plant Dis. 76:348-352.

Turechek, W. W., and Köller, W. 2004. Managing resistance of Venturia inaequalis to the strobilurin fungicides. Online publication. Plant Health Prog. doi.org/ 10.1094/PHP-2004-0908-01-RS 